\title{
La empagliflozina disminuye la mortalidad cardiovascular y el riesgo de hospitalización en pacientes con insuficiencia cardíaca
}

\author{
Empagliflozin decreases cardiovascular mortality and the risk of hospitalization in heart failure patients
}

\section{Comentado de:}

Packer M, et al. N Engl J Med. 2020;383(15):1413-1424. PMID: 32865377. doi: 10.1056/NEJMoa2022190 ${ }^{1}$

\section{Objetivo}

Determinar si la empagliflozina, un inhibidor del cotransportador de sodio-glucosa tipo 2 (SGLT-2), disminuye el riesgo de presentar eventos cardiovasculares y renales en pacientes con insuficiencia cardíaca crónica (ICC) y fracción de eyección del ventrículo izquierdo (FEVI) disminuida, con o sin diabetes.

\section{Diseño, lugar y población}

Ensayo clínico multicéntrico, aleatorizado, controlado con placebo y doble ciego, realizado en 520 instituciones de 20 países de distintos continentes.

Fueron incluidos pacientes mayores de 18 años con ICC y FEVI de $40 \%$ o menor, clase funcional de II a IV de la Escala New York Heart Association (NYHA) y tratamiento médico optimizado. Los pacientes con $\mathrm{FEVI}$ mayor a $30 \%$ debían además haber sido hospitalizados por insuficiencia cardíaca (IC) en los doce meses previos al ingreso al estudio o poseer niveles elevados de la porción $\mathrm{N}$-terminal del pro-péptido natriurético tipo B (NT-proBNP, por sus iniciales en inglés).

\section{Intervención}

Los pacientes fueron asignados aleatoriamente en proporción $1: 1$ a dos ramas de investigación, con estratificación según la región geográfica. Los pacientes del grupo intervención recibieron 10 mg por día de empagliflozina, y en ambos grupos fue administrada la medicación habitual para la ICC.

\section{Medición de resultados principales}

El desenlace principal compuesto -mortalidad de causa cardiovascular (CV) y hospitalización por IC- fue analizado como tiempo al primer evento.

De manera secundaria, fueron registradas las hospitalizaciones totales por IC (primera y recurrentes), el deterioro de la tasa de filtrado glomerular (TFG) y un resultado renal compuesto (diá- lisis crónica, trasplante renal o reducción sostenida de la TFG).

Los pacientes fueron evaluados cada dos o tres meses. La TFG fue evaluada de 23 a 45 días después de la descontinuación del tratamiento activo o el placebo. Los autores realizaron un análisis por intención de tratar.

\section{Resultados Principales}

Fueron incluidos 3.730 pacientes: 1.863 en el grupo intervención y 1867 en el grupo placebo. La mediana de seguimiento fue de 16 meses.

En la Tabla 1 se presenta el desenlace principal compuesto y disgregado. Se puede observar que el desenlace compuesto ocurrió con mayor frecuencia en el grupo placebo, y que esta diferencia fue atribuible, principalmente, a las hospitalizaciones por IC. EI número necesario de pacientes a tratar con empagliflozina para prevenir un evento primario fue de 19 (intervalo de confianza [IC] del $95 \% 13$ a 37), sin diferencia entre los pacientes con y sin diabetes.

En comparación con el grupo de participantes que recibió placebo, en el grupo empagliflozina se documentó una menor incidencia de hospitalizaciones totales (primera y recurrentes) por IC (388 vs 553, cociente de riesgos [HR] 0,70; IC $95 \% 0,58$ a 0,85, $\mathrm{p}<0,001)$, una mayor lentitud en la disminución de la TFG estimada $\left(-0,55\right.$ vs. $-2,28 \mathrm{~mL}$ por minuto por $1,73 \mathrm{~m}^{2}$ del área de superficie corporal por año, $p<0,001)$. La infección no complicada del tracto genital fue más frecuente en el grupo empagliflozina.

\section{Conclusiones}

Entre los pacientes aleatorizados a empagliflozina, el riesgo de muerte cardiovascular u hospitalización por insuficiencia cardíaca fue menor que en los del grupo placebo, de manera independiente de la presencia o la ausencia de diabetes mellitus.

Fuente de financiamiento / Conflicto de interés de los autores: El estudio fue financiado por Boehringer Ingelheim y Eli Lilly. Todos los autores completaron la declaración de conflictos de interés y reportaron haber recibido honorarios personales y/o subsidios de diversas empresas farmacéuticas durante o fuera de la conducción de este estudio.

Tabla 1. Comparación de los resultados principales entre ambos grupos. Notas: ${ }^{a}$ incidencia de eventos/100 pacientes-año de seguimiento; IC: intervalo de confianza

\begin{tabular}{|l|c|c|c|}
\hline Desenlace & $\begin{array}{c}\text { Grupo empagliflozina, } n^{a}(\%) \\
\mathrm{N}=1.863\end{array}$ & $\begin{array}{c}\text { Grupo placebo, } \mathrm{n}^{a}(\%) \\
\mathrm{N}=1.867\end{array}$ & Hazard ratio (IC 95\%) \\
\hline Resultado principal compuesto & $361(15,8)$ & $462(21)$ & $0,75(0,65 \mathrm{a} 0,86)$ \\
\hline Mortalidad cardiovascular & $187(7,6)$ & $202(8,1)$ & $0,92(0,75$ a 1,12$)$ \\
\hline Hospitalización por insuficiencia cardíaca & $246(10,7)$ & $342(15,5)$ & $0,69(0,59$ a 0,81$)$ \\
\hline
\end{tabular}




\section{Comentario}

Investigaciones de buena calidad metodológica realizadas en los últimos años con los nuevos hipoglucemiantes orales han puesto en evidencia que estas drogas producen un efecto positivo en los sistemas cardiovascular y renal, disminuyendo tanto la mortalidad como la progresión de la enfermedad ${ }^{2}$.

Los inhibidores del SGLT-2 están actualmente recomendados como primera o segunda línea terapéutica en los pacientes con diabetes y enfermedad cardiovascular ${ }^{3,4}$ o con enfermedad renal crónica establecida ${ }^{5,6}$, quedando planteado el interrogante sobre su utilidad en personas con IC avanzada y/o enfermedad renal ${ }^{7}$. Los resultados de este ensayo esta población son alentadores, ya que documentó una reducción del $31 \%$ en las hospitalizaciones por IC con la administración de empagliflozina en comparación con el uso de placebo, además de un enlentecimiento de la caída de la TFG que se acompaña de un menor riesgo de desenlaces renales de gravedad.

Sin embargo, un aspecto importante a tener en cuenta es su alto costo y que, al menos en Argentina, todavía no están cubiertos por los programas para pacientes con diabetes, lo que dificulta su utilización a largo plazo en las pacientes con enfermedades crónicas.

\section{Conclusiones de la comentadora}

A partir de los resultados favorables observados en este ensayo, recomendamos considerar el uso de la empagliflozina en aquellos pacientes que desarrollan IC avanzada sumado al tratamiento habitual de esta entidad, independientemente de la presencia o la ausencia de diabetes.

Natalia Jimena Ponce de León [ Servicio de Medicina Familiar y Comunitaria, Hospital Italiano de Buenos Aires .natalia.poncedeleon@hospitalitaliano. org.ar ]

Ponce de León N. La empagliflozina disminuye la mortalidad cardiovascular y el riesgo de hospitalización en pacientes con insuficiencia cardíaca. Evid Actual Pract Ambul. 2021;24(4):e002159. Available from: https://dx.doi.org/10.51987/EVIDENCIA.V25I1.6976. Comentado de: Packer M, et al. Cardiovascular and Renal Outcomes with Empagliflozin in Heart Failure. N Engl J Med. 2020;383(15):1413-1424. PMID: 32865377. doi: 10.1056/NEJMoa2022190

\section{Referencias}

1. Packer F, Anker SD, Butler J, et al. Cardiovascular and Renal Outcomes with Empagliflozin in Heart Failure. N Eng J Med. $2020 ; 383(15): 1413-1424$. Available from: 10.1056/NEJMoa2022190.

2. Neuen BL, Cherney DZ, Jardine MJ, et al. Sodium-glucose cotransporter inhibitors in type 2 diabetes: thinking beyond glucose lowering. . CMAJ. 2019;191(41):1128-1135. Available from: 10.1503/cmaj.190047.

3. Argentina. Ministerio de Salud. Dirección Nacional de abordaje Integral de enfermedades no transmisibles. Guía de Práctica Clínica Nacional sobre Prevención, Diagnóstico y Tratamiento de la diabetes mellitus tipo 2 (DM2); 2019. Available from: https://bancos.salud.gob.ar/sites/default/files/202009/guia-nacional-practica-clinica-diabetes-mellitius-tipo2_2019.pdf.

4. Bailon T, Cena K, Rau S, et al. Nueva guía de diabetes mellitus tipo 2 en Argentina. Comentado de: Argentina. Ministerio de Salud. Dirección Nacional de Abordaje Integral de Enfermedades No Transmisibles. Guía de Práctica Clínica Nacional sobre Prevención, Diagnóstico y Tratamiento de la Diabetes Mellitus Tipo 2 (DM2) 2019. Evid Actual Pract Ambul. 2020;23(4):e002107. Available from: 10.51987/evidencia.v23i4.6906;http: //www.evidencia.org/index.php/Evidencia/article/view/6906.

5. Vandvik S, Lytvyn PO, Guyatt L, et al. SGLT-2 inhibitors or GLP-1 receptor agonists for adults with type 2 diabetes: a clinical practice guideline. BMJ. 2021;373:1091. Available from: 10.1136/bmj.n1091.

6. Davies MJ, D'Alessio DA, Fradkin J, et al. Management of hyperglycaemia in type 2 diabetes, 2018. A consensus report by the American Diabetes Association (ADA) and the European Association for the Study of Diabetes (EASD). Diabetologia. 2018;61(12):2461-2498. Available from: 10.1007/ s00125-018-4729-5.

7. Mcmurray JJV, Solomon SD, Inzucchi SE, et al. Dapagliflozin in patients with heart failure and reduced ejection fraction. $\mathrm{N}$ Engl $\mathrm{J}$ Med. 2019;381(21):1995-2008. Available from: 10.1056/NEJMoa1911303. 